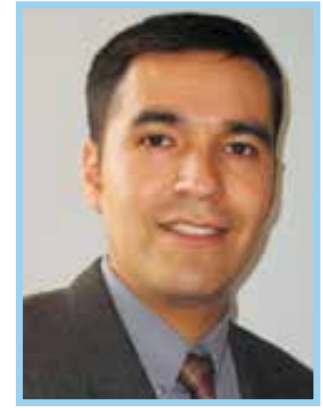

David Parra Reyes

\section{EL LENGUAJE EN LOS NIÑOS CON PARÁLISIS CEREBRAL}

\section{RESUMEN}

El niño con parálisis cerebral (PC) se caracteriza por la dificultad motriz en la realización del lenguaje oral, manifestando desde alteraciones lingüísticas leves hasta la más absoluta imposibilidad para la expresión oral. En general, los niños con PC van a presentar alteraciones tanto en la adquisición como en el desarrollo en los niveles del lenguaje y problemas motores de expresión que afectan la producción del habla y de la voz.

\section{PALABRAS CLAVE}

Parálisis cerebral infantil, lenguaje, alteraciones del lenguaje, habla.

\section{ABSTRACT}

A child with cerebral palsy (CP) is characterized by the movement problems in the spoken language practice, showing since mild linguistic alterations to the impossibility for spoken expression. In general, children with PC show alterations in the acquisition as well as in language levels and expression movement problems that affect the speech and voice production.

\section{KEYWORDS}

Child cerebral palsy, language, language alterations, speech.

\section{INTRODUCCIÓN}

$\mathrm{U}$ n niño con PC puede manifestar retrasos en el lenguaje, trastornos de la articulación, respiración, fonación, prosodia y fluencia en distintos grados. Las repercusiones lingüísticas dependerán del tipo de alteración y de la gravedad de la misma. Se calcula que alrededor del 60 al 80\% de estos niños presentan algún grado de afectación en su lenguaje, siendo en las tetraparesias donde se observa el porcentaje más elevado de trastornos del lenguaje.

Los problemas en la adquisición del lenguaje varían mucho de un niño a otro, según el alcance de la lesión a nivel motriz, y la posible incidencia de trastornos asociados (capacidades cognitivas, sensoriales, perceptivas, etc.).

En el tema del desarrollo cognitivo y motor de los niños con parálisis cerebral, se observa comúnmente una fuerte relación entre el lenguaje como función cognitiva, con el funcionamiento de las estructuras fonoarticulatorias en cuanto al aspecto motor. En este sentido, el lenguaje es dividido en emisión y recepción, Lacerda (2003) refiere que el proceso del habla como una forma personal y final del lenguaje, como es la expresión oral (enfatizando a la motricidad como ítem fundamental de este acto). Agrega, también, la importancia del medio social como un factor imprescindible para el desarrollo lingüístico, y con ello, el desarrollo de la cognición, entre ellas el lenguaje. Finnie (1999) menciona que el desarrollo del lenguaje se 
relaciona con los mecanismos motores del habla; sin embargo, más allá de esta perspectiva, Connor y Cols. (1978) observan el lenguaje del niño con parálisis cerebral con un punto de vista piagetiano. Refieren que los mecanismos previos al habla, el lenguaje y la cognición son aspectos fundamentales del desarrollo del niño, mostrando la importancia de la relación entre lenguaje y desarrollo cognitivo. De este modo, existen dos factores que influenciaran la calidad y la prontitud del desarrollo cognitivo de un niño: la capacidad de interactuar con el medio y el ambiente que lo rodea.

\section{Desarrollo del lenguaje en niños con PC}

Durante los tres primeros años de vida se sientan las bases de la comunicación a través de las interacciones del niño con los padres (entorno social y familiar). El niño con PC tiene dificultades para mantener la mirada, coordinar los movimientos oculares para seguir un objeto, desplazar la cabeza para el seguimiento de un objeto, coordinar atención ocular y sonido (tiempo de reacción), realizar la emisión vocal en el momento deseado, seguir turnos de intervención a causa de su lentitud, manipular los objetos relacionados con lo que se está comunicando (juguetes, utensilios de comida, etc.), utilizar coordinadamente los elementos habituales propios de una comunicación (posición del cuerpo, mirada, capacidad de vocalización, etc.), el balbuceo es más pobre en diversidad y frecuencia que el del niño con un desarrollo normal, se altera la aparición e inhibición de los reflejos orales, el dominio de las praxias orales es tardío e incompleto, la limitación de los movimientos del cuerpo alteran el desarrollo y la funcionalidad de la laringe, etc.

Sinclair (1970) describe el desarrollo lingüístico y cognitivo dentro las teorías de Piaget, en donde, además, lo relaciona con los trastornos lingüísticos en el niño con PC, que se caracteriza por el retraso en la adquisición del lenguaje y del desarrollo cognitivo. Sinclair, se preocupa en los aspectos de imitación, juego motriz, juegos simbólicos y la exploración de objetos.

El lenguaje del niño con PC va más allá de la emisión y la recepción. Este lenguaje debe ser conocido como consecuencia de un momento anterior, además sin la presencia del simbolismo, que se da durante el periodo sensorio-motor.

Lacerda enfatiza, además, que el lenguaje del niño con PC, debe ser ubicado dentro de su desarrollo cognitivo, percibiendo como ocurre el proceso de comunicación entre el niño y el medio, a pesar de su limitación sensorio - motor.

Existe la necesidad de estimular al niño interactuar con el medio, favoreciendo un intercambio activo de experiencias con su ambiente. En consecuencia, el niño con PC, tendría una oportunidad de vivenciar las construcciones del periodo sensorio-motor, enriqueciendo así su lenguaje.

Piaget (1980) menciona que la acción y fuente del acto inteligente, son las experiencias sensoriomotoras básicas y para un desarrollo cognitivo normal.

Analizando esa afirmación, se puede observar que los niños que se desarrollan con deficiencias físicas, tendrían un déficit cognitivo causado por la limitación o restricción de movimiento en el ambiente y de manipulación de los objetos.

En complemento, Piaget, Young (1991) concluyen que los niños con PC, así como los esquemas sensorio-motores, y los procesos perceptivos estarían perjudicados, lo que llevaría en un retardo o un inadecuado pensamiento representativo y del simbolismo, afectando su desarrollo del lenguaje.

El niño con PC manifiesta una deficiencia en el desarrollo cognitivo, no siendo esto una regla general en todos los niños con PC, debido a las alteraciones motrices que limitan las experiencias sensorio-motoras. Finnie (1999) afirma, que por la falta de exploración motora del niño con PC, sus experiencias de lenguaje 
son menores, destacando que las personas que forman parte de su medio en que el niño vive, deben favorecer a que las experiencias que llegue al niño tengan sentido para que puedan ser aprovechadas por él.

Braga (2005) afirma que en las formas graves de parálisis cerebral presentan movimientos involuntarios, mayor dificultad de agarrar los objetos debido a sus condiciones motoras; sin embargo, no están limitados a la intensión de acción, control cognitivo, representación consciente, atención, motivación y relación social.

Así, este niño con PC grave puede planificar su acción y lo ejecuta a través de otras personas, en el caso que impliquen movimiento o contacto físico con el objeto.

Vygotsky (1993), admite que las leyes que fundamentan el desarrollo son las mismas para niños con o sin deficiencias. Las diferencias serían los caminos que escogen para que estos niños puedan procesar el mundo, incrementando así las funciones de desarrollo cognitivo del niño que aparecen primero en el plano social y, luego, en lo psicológico.

Cole (1989), concibe lo social y lo individual como un sistema de interacción único. Es así que la relación que se establece entre el niño con PC con las personas con buena capacidad motora, puede ser un elemento fundamental para su desarrollo. La acción compartida estimularía la capacidad del niño a establecer vínculos sociales que favorecería a su desempeño cognitivo.

Braga afirma también, que la deficiencia no es el motor propulsor de los nuevos caminos que el niño con PC tiende a buscar. Son las dificultades derivadas de ese problema motor y sus repercusiones sociales que la llevan a la búsqueda de nuevos caminos. Para Vygotsky, el futuro de estos niños dependerá mucho de las posibilidades que ellas vean al tener en la interacción con el medio.
Braga complementa que las relaciones sociales establecidas por los niños se dan a través de símbolos que hacen parte de un sistema cultural. En el caso del deficiente, no es el tipo de instrumento o el signo que es utilizado, sino el significado que desea representar que tiene mayor importancia. La función primordial es interiorizar el lenguaje y desarrollar funciones mentales que tienen significado como base.

El niño construye y orienta su proceso de desarrollo cognitivo a través de la motivación, de la atención y de la acción. Un niño con PC no tiene limitaciones relativas a la motivación y a la atención. El bloqueo solo se limita a la acción. Esta acción se determina como un comportamiento consciente y con propósito orientado hacia un objetivo, siendo su aspecto motor y táctil secundario y hasta irrelevante.

Principalmente, aparecen la génesis social, la intencionalidad, la planificación, la definición de objetivos y la motivación. En este fundamento, el niño con PC se halla en un énfasis cognitivo, a partir de sus motivaciones, tal como propone Vygotsky, y orienta su desarrollo con base en la atención, motivación y acción interna, como propone Piaget.

Según Braga (2005), bastaría que el acto motor fuese ejercido por otro integrante del medio, un mediador. Todo el potencial cognitivo del niño fue usado en la construcción mental de la tarea y lo orienta, dirigiendo la tarea para ese mediador a partir de sus propias perspectivas. Ese mediador es considerado como de creador conjunto. $\mathrm{Su}$ función es de cooperación, una operación en conjunto favoreciendo una construcción recíproca.

El niño y el mediador se van modificando en el proceso de interacción. Ambos desarrollan un proceso creativo para el descubrimiento de nuevas rutas de comunicación y acción con su medio. 
Dimensiones del lenguaje alterados en los niños con parálisis cerebral

En la parálisis cerebral se constatan dificultades en las diferentes dimensiones del lenguaje:

> En la forma o el aspecto externo de lo que se habla (fonología, morfología y sintaxis).

, En el contenido o de lo que se habla (semántica).

, En el uso o la función o utilidad que hablar tiene para quienes lo hacen (pragmática).

En el niño con PC la adquisición del repertorio fonológico es más lento (déficit en el control neuromuscular), aunque sigue las mismas fases del desarrollo normal. Generalmente, los niños con parálisis cerebral atetósica presentan capacidades fonológicas inferiores a los espásticos.

En estos niños existe cierta tendencia a reducir la longitud media de los enunciados, así como su complejidad sintáctica (utilizan enunciados cortos y sencillos, adaptando el discurso a la dificultad motriz que presentan). La dificultad motriz hace que el niño, a pesar de poseer el nivel psicolingüístico adecuado, utilice expresiones sintácticas muy limitadas.

Las dificultades que manifiesta el niño con parálisis cerebral a nivel semántico se fundamentan en la mayoría de los casos en un léxico muy limitado y en unas relaciones semánticas muy pobres. Estos niños priorizan el uso de nombres, verbos y adjetivos sobre el uso de pronombres y adverbios.

La pobreza en la expresión oral, tanto lingüística como cognitiva es consecuencia de las limitaciones experienciales (el vocabulario se desarrolla en función de las experiencias que el niño tiene, pero la falta de control postural y los movimientos limitados, limitan que experimente determinadas cosas) y del déficit motor (utilizan un léxico más fácil desde un punto de vista motriz).

En el uso del lenguaje (pragmática) se constatan dificultades desde los primeros momentos. El niño con parálisis cerebral tiene problemas para iniciar conversaciones, dispone de menos ocasiones para utilizar el lenguaje, mostrar desacuerdo, buscar o seleccionar un determinado interlocutor, etc.

En el niñocon PC, la producción dellenguaje varía de un caso a otro, dependiendo de si el problema motor afecta a uno o a todas las estructuras y funciones implicadas en la expresión oral.

\section{Alteraciones que interfieren con el desarrollo de su lenguaje en el niño con PC}

Las dificultades más frecuentes en este niño con PC que interfieren con el desarrollo de su lenguaje son las siguientes: 


\begin{tabular}{|c|c|c|c|}
\hline Alteraciones & Características & Dificultades & Interferencia en el lenguaje \\
\hline $\begin{array}{l}\text { Tono } \\
\text { muscular y en } \\
\text { la postura }\end{array}$ & $\begin{array}{l}\text { Tono muscular } \\
\text { excesivamente } \\
\text { alto, bajo o } \\
\text { fluctuante. }\end{array}$ & $\begin{array}{l}\text { En el control de } \\
\text { los movimientos } \\
\text { de la cabeza o del } \\
\text { tronco, espasmos } \\
\text { o contracciones } \\
\text { excesivas de los } \\
\text { músculos de la nuca o } \\
\text { del cuello. }\end{array}$ & $\begin{array}{l}\text { - Los patrones reflejos pueden } \\
\text { aparecer en el momento que el } \\
\text { niño intenta hablar, dificultando } \\
\text { la emisión. } \\
\text { - Imposibilidad de hacer } \\
\text { movimientos fonoarticulatorios } \\
\text { diferenciados. } \\
\text { - Descontrol y bloqueo de } \\
\text { movimientos del cuerpo cuando } \\
\text { intenta hablar. }\end{array}$ \\
\hline Mímica facial & $\begin{array}{l}\text { - Movimientos } \\
\text { lentos y } \\
\text { limitados. } \\
\text { - En otros casos } \\
\text { exagerada, } \\
\text { con gestos } \\
\text { bruscos y } \\
\text { asimétricos. }\end{array}$ & $\begin{array}{l}\text { Mímica fija, que } \\
\text { permanece en una } \\
\text { misma posición, } \\
\text { resultando inapropiado } \\
\text { para el contexto } \\
\text { comunicativo en el que } \\
\text { interaccionan. }\end{array}$ & $\begin{array}{l}\text { Durante los primeros meses (el } \\
\text { niño sonríe poco, tiene siempre la } \\
\text { misma expresión, o hay una escasa } \\
\text { respuesta a los diferentes estímulos } \\
\text { de sus familiares). }\end{array}$ \\
\hline $\begin{array}{l}\text { Articulación } \\
\text { irregular, } \\
\text { imprecisa o } \\
\text { distorsionada } \\
\text { (disartria) }\end{array}$ & $\begin{array}{l}\text { - Dificultades } \\
\text { en la } \\
\text { movilidad } \\
\text { de la lengua, } \\
\text { labios o } \\
\text { maxilares. } \\
\text { - Según el } \\
\text { caso, el tono } \\
\text { muscular de la } \\
\text { lengua puede } \\
\text { ser muy alto o } \\
\text { bajo. }\end{array}$ & $\begin{array}{l}\text { En algunos casos } \\
\text { la dificultad es tal } \\
\text { que deberíamos } \\
\text { hablar de anartria } \\
\text { (imposibilidad para } \\
\text { articular correctamente } \\
\text { todos o casi todos los } \\
\text { fonemas). }\end{array}$ & $\begin{array}{l}\text { - El niño presenta dificultades } \\
\text { para ejecutar los movimientos } \\
\text { necesarios para articular } \\
\text { correctamente los fonemas. } \\
\text { - Los movimientos de su lengua } \\
\text { son imprecisos e inadecuados en } \\
\text { fuerza. } \\
\text { - En ocasiones, incluso los } \\
\text { movimientos son involuntarios. }\end{array}$ \\
\hline $\begin{array}{l}\text { Los reflejos } \\
\text { orales }\end{array}$ & $\begin{array}{l}\text { Pueden no } \\
\text { aparecer o por } \\
\text { el contrario, } \\
\text { no desaparecer } \\
\text { hasta edades } \\
\text { muy avanzadas. }\end{array}$ & $\begin{array}{l}\text { Algunos niños no } \\
\text { tienen el reflejo de } \\
\text { succión en los primeros } \\
\text { meses o no inhiben } \\
\text { el de morder, con } \\
\text { lo que se retrasa la } \\
\text { masticación. }\end{array}$ & $\begin{array}{l}\text { Dificultad en el proceso de la } \\
\text { alimentación, y en la realización de } \\
\text { las praxias de la zona oral. }\end{array}$ \\
\hline
\end{tabular}




\begin{tabular}{|c|c|c|c|}
\hline Alteraciones & Características & Dificultades & Interferencia en el lenguaje \\
\hline Babeo & $\begin{array}{l}\text { Retención de } \\
\text { saliva en la } \\
\text { cavidad oral } \\
\text { (sialorrea). }\end{array}$ & $\begin{array}{l}\text { - Limitación para } \\
\text { deglutir la saliva. } \\
\text { - Disminución } \\
\text { propioceptiva de la } \\
\text { sensibilidad oral. }\end{array}$ & Dificultad en el habla. \\
\hline $\begin{array}{l}\text { Respiración } \\
\text { oral }\end{array}$ & $\begin{array}{l}\text { - Respiración } \\
\text { superficial } \\
\text { o mal } \\
\text { coordinada e } \\
\text { irregular. } \\
\text { - También } \\
\text { pueden } \\
\text { aparecer } \\
\text { espasmos en } \\
\text { el diafragma. }\end{array}$ & $\begin{array}{l}\text { - No controlan el flujo } \\
\text { de aire, por lo que a } \\
\text { menudo lo agotan al } \\
\text { ir a hablar, o incluso } \\
\text { hacen coincidir la } \\
\text { inspiración con el } \\
\text { intento de fonación. } \\
\text { - Asincronía entre } \\
\text { la inspiración y } \\
\text { espiración y también } \\
\text { de los movimientos } \\
\text { laríngeos. }\end{array}$ & $\begin{array}{l}\text { - Esto influye en el volumen y } \\
\text { articulación de la voz, y provoca } \\
\text { alteraciones en la fluencia del } \\
\text { lenguaje y en la prosodia. } \\
\text { - Aparecen pausas inadecuadas y se } \\
\text { altera la entonación, el ritmo y la } \\
\text { melodía. } \\
\text { - Unas veces el habla es muy } \\
\text { explosiva y otras veces presenta } \\
\text { variaciones excesivas de ritmo. }\end{array}$ \\
\hline $\begin{array}{l}\text { Alteraciones } \\
\text { de la voz } \\
\text { (intensidad, } \\
\text { timbre, etc.) }\end{array}$ & $\begin{array}{l}\text { Consecuencia de } \\
\text { los déficits en el } \\
\text { control postural, } \\
\text { el tono y la } \\
\text { fuerza muscular. }\end{array}$ & $\begin{array}{l}\text { - Cualquier emisión } \\
\text { del niño sin un } \\
\text { adecuado control del } \\
\text { cuello y de la cabeza } \\
\text { o sin la suficiente } \\
\text { relajación del tono } \\
\text { muscular ocasionará } \\
\text { trastornos en la voz. } \\
\text { - Por el mal control del } \\
\text { velo del paladar. } \\
\text { - Cierre incompleto } \\
\text { de la entrada } \\
\text { palatofaríngea. }\end{array}$ & $\begin{array}{l}\text { Presencia de hipernasalidad } \\
\text { constante o intermitente al emitir } \\
\text { sonidos o palabras. }\end{array}$ \\
\hline
\end{tabular}




\section{Alteraciones en la expresión oral según tipo de parálisis cerebral}

Según el tipo de parálisis cerebral las alteraciones en la expresión oral suelen variar, como por ejemplo:

, En la parálisis cerebral espástica (el $70 \%$ de los casos), las manifestaciones más importantes son la espasticidad y la hipertonía. La debilidad de los movimientos voluntarios afecta a los labios y a la lengua. La lengua, torpe e hipertónica, no puede extenderse más allá de los dientes. La lengua puede estar desviada o con diferente tono a un lado que al otro. Los movimientos de los labios son lentos y limitados. El rostro del niño es inexpresivo y no tiene control del babeo. La articulación es lenta (vocales distorsionadas e imprecisión en la articulación de consonantes). Hay escape nasal, fallos en el ritmo y espasticidad en los músculos respiratorios. Se produce hipertonía de las cuerdas vocales, tono bajo, voz forzada, interrupciones, brevedad en las frases que producen y lentitud en el habla. La voz es monótona, sin entonación, y a menudo comienza la emisión con un espasmo. Estos niños tienen dificultad en la construcción de frases.

> En la parálisis cerebral atetósica, se produce afectación en la lengua, en los músculos de la masticación y en la respiración. La articulación de los fonemas se interfiere por las muecas de la cara y los movimientos involuntarios de la lengua (fluctuaciones bruscas del tono muscular). La voz es fuerte en exceso, jadeante y monótona, a consecuencia de las contracciones espasmódicas irregulares del diafragma y otros músculos de la respiración. Presentan dificultades en el ritmo, y el curso de la palabra es variable de un momento a otro, deteriorándose en mayor medida ante determinadas situaciones emocionales.
> En la parálisis cerebral atáxica, la voz tiende a ser baja, monótona, con interrupciones y con excesivas variaciones en intensidad. La articulación de los fonemas es lenta, debido a los movimientos torpes e imprecisos de la lengua. Discrimina con imprecisión en la articulación de las consonantes y presencia de habla escandida.

> En la parálisis cerebral mixta, se producen alteraciones de los movimientos de labios, lengua y paladar, por lo que la articulación de consonantes es imprecisa. Asimismo, se caracterizan por hipernasalidad y variaciones en la prosodia. Con frecuencia hay movimientos de protusión lingual y dificultades en la deglución.

\section{CONCLUSIONES}

Las fases del desarrollo cognitivo son las mismas para todos los niños y funcionan como pre requisitos de manera secuencial. Así, las informaciones que ya habían sido almacenadas después de una ejecución, sufren una perfección después de una nueva ejecución, es así que el niño se va desarrollando y adquiriendo destrezas importantes para su desempeño, estos son los hitos de desarrollo.

Un niño con PC demora en algunos aspectos de ese proceso, que no representan dificultades para los niños en otras condiciones. Su desarrollo motor es inferior, en algunos casos es marcado, en otros, no tanto, pero ese cuadro lo imposibilita de almacenar datos de una acción, como los otros niños. Las experiencias se tornan más limitadas. Observando las experiencias como fundamentales para el desarrollo de la cognición, y teniendo el lenguaje como objetivo principal, ese niño precisa crear medios que lo lleven a superar esos obstáculos y mostrar que él es apenas diferente. El niño cuyo desarrollo está perjudicado por una limitación no es un niño mejor desarrollado que los demás; sino que se desarrolla de una forma especial y lenta. 
En ese sentido, prima la necesidad de aprender a observar y entender la manera de actuar de ese niño, para luego poder clasificar su lenguaje. Del mismo modo, sin verbalizar, él logra transmitir su lenguaje, una mirada, una sonrisa son indicios comunicativos. Cabe mencionar que los que conviven con ese niño deben interpretar sus tentativas de interacción, para facilitarlas y responder a ellas. Además, que nadie se comunica solo. Es necesario la interacción, el que comunica y alguien que recibe el mensaje. En los casos de parálisis cerebral es preciso un paso demás, el de descifrar la forma como el mensaje fue trasmitido.

Entender el lenguaje del niño con PC es percibir el modo como él se comunica con su medio a pesar de su compromiso motor. Se debe ubicar sus intenciones comunicativas dentro de su desarrollo cognitivo, propiciando un cambio activo de ese niño con el mundo que lo rodea y favoreciendo que vivencie sus construcciones correspondientes a su periodo de vida. De ese modo, ocurre el enriquecimiento del lenguaje.

\section{REFERENCIAS}

Ault, R. (1998). Desarrollo cognitivo del niño: la teoría de Piaget y el abordaje del proceso. Río de Janeiro: Zahar Editores.

Bobath, K. (1994). Una base neurofisiológica para el tratamiento de la parálisis cerebral. São Paulo: Editora Manole.

Braga, L. (2005). Cognição e paralisia cerebral: Piagete Vygotsky emquestão. Salvador: Sarah letras.

Cruickshank, W. \& Raus, G. (2005). Cerebral palsy: it's individual and community problems. California: Syracuse University Press.

KEATS, S. (1970). Cerebral palsy. Illinois: Thomas Books Publisher.
Este artículo también, me permite abrir nuevos horizontes, llevando estos conceptos y conocimientos a los profesores de instituciones educativas especiales para que así puedan ayudar en la nivelación de los alumnos portadores de parálisis cerebral. Considerando que estos niños deberían estar en aulas donde todos estén en una misma etapa de desarrollo. Cada vivencia sería importante ya que permitiría estimularse y aprender de las habilidades desarrolladas del otro niño.

Tener conocimientos sobre cognición es fundamental para lidiar con el lenguaje y llegar, hasta el aprendizaje. De una forma u otra, las etapas del desarrollo cognitivo son vencidas con la superación de la anterior por el aprendizaje. Mucho más que limitar este tema a las escuelas especiales, se pretende que esto se lleve a las escuelas básicas y regulares, principalmente a los "nidos" y niveles básicos como los "jardines". En la fase de la enseñanza infantil, recibir la correcta estimulación es fundamental.

Lacerda, E. (2003). Reflexões sobre a terapia fonoaudiológica da criança paralítico-cerebral. São Paulo: Memnon.

Lefèvre, A. (2010). Neurología infantil: semiología, clínica y tratamiento. São Paulo: Sarvier.

Leitão, A. (2003). Parálisis cerebral: diagnóstico, terapia y rehabilitación. Río de Janeiro: Atheneu.

Lima, B. (2006). LinguagemepensamentoemPiaget:conseqüências metodológicas para o ensino de línguas. Petrópolis: Vozes.

Mueller, H. (1999). Fala. In: Finnie, N. A - O manuseioem casa da crianza compara lisia cerebral. São Paulo: Manole. 
Piaget, J. (1961). El lenguaje y el pensamiento del niño. Río de Janeiro: Fundo de Cultura.

Seber, M. (2009). Construção da inteligência pela criança. São Paulo: Scipione, pp. 13-35 (série Pensamento e ação no magistério).

Tabith Jr. (1995). Foniatría: disfonías, fisuras labio palatinas, parálisis cerebral. São Paulo: Cortez.
Vygotsky, L. (1964). Pensamiento y lenguaje. Buenos Aires: Editorial Lautaro.

Zorzi, J. (1999). A intervenção fonoaudiológica nasalterações da linguagem infantil. Río de Janeiro: Revinter.

Zorzi, J. (1994). Linguagem e desenvolvimento cognitivo: a evolução do simbolismo nacriança. São Paulo: Pancast Editora. 